\title{
On the Input-Output Relationship for Molecular Communications in General First-Order Chemical Reaction-Diffusion Systems
}

\author{
Malcolm Egan \\ Univ. Lyon, INSA Lyon, INRIA, CITI \\ Villeurbanne, France
}

\author{
Bao Quoc Tang \\ Institute of Mathematics and \\ Scientific Computing \\ Univ. Graz \\ Graz, Austria
}

\author{
Bayram Cevdet Akdeniz \\ Univ. Lyon, INSA Lyon, INRIA, CITI \\ Villeurbanne, France
}

\begin{abstract}
Any general model for a molecular communication system must account for both chemical reactions and diffusion. In particular, the chemical reactions capture processes of information molecule production and detection as well as inter-symbol mitigation methods in the channel. A popular model for such reaction-diffusion systems is the reaction-diffusion master equation. In this paper, we study the statistics for the number of molecules at the receiver, yielding a stochastic input-output relationship. Our focus is on a general class of first-order reactions and linear diffusion. This class of models admits a stationary distribution for the long-term behavior. We obtain a simple Gaussian approximation for this stationary distribution by exploiting recent results characterizing the underlying deterministic reaction-diffusion system. We prove exponential rates of convergence in the underlying deterministic system and verify our approximation via stochastic simulation.
\end{abstract}

\section{INTRODUCTION}

In complex biochemical processes, both chemical reactions and diffusion play a fundamental role. This is also true in the context of molecular communications, where reactions model the information molecule production and detection processes while diffusion models the transfer of molecules from the transmitter to the receiver [10]. Reactions have also been shown to provide a means of improving the reliability of communication by reducing inter-symbol interference [12].

A popular model for chemical reaction-diffusion systems is the reaction-diffusion master equation (RDME) [11]. In this stochastic model, the evolution of the number of each chemical species in each location is governed by a continuous-time Markov chain. As a consequence, the probability distribution for the system state is described by a coupled system of differential equations.

In general, the RDME model can only be solved numerically using stochastic simulation methods. While there are now many efficient methods based on the simulation of continuous-time Markov processes (e.g., the work of Gillespie [8]), it remains a challenge to obtain simple characterizations of the input-output relationship for the purpose of designing modulation and detection schemes.

One solution to this problem is to exploit continuous-time filters, such as in the recent work of Chou [3,4]. An alternative approach is to exploit the structure of the RDME. In particular, when the chemical reactions admit reversibility properties, then an equilibrium state is often reached for sufficiently large sampling times. This state can be exploited to implement simplified detection schemes, as discussed in the case of a chemical reaction networks model in [5]. This initial study of encoding information in the equilibrium state also suggests that aside from the simplified detection, feedback from the receiver to the transmitter is built into the approach.

In this paper, we study molecular communication systems modeled by the RDME in the case of general first-order chemical reactions. In order to capture the fact that not all chemical species act as information molecules-e.g., they are involved only in information molecule production or detection processes-we also consider spatially inhomogeneous diffusion coefficients.

Our focus is on characterizing the input-output relationship for this class of RDME models, which provides the connection between the initial quantities of molecules in the transmitter and the quantities at time $t$ in the receiver. This input-output relationship yields the output statistics for the RDME model, which can be exploited to develop new modulation and detection schemes such as equilibrium signaling [5].

Of particular interest is the behavior of the RDME model for large sampling times. In practice, many biochemical oscillators have large periods [14] which makes it difficult to sample at short time intervals. As a consequence, it is desirable to develop detection strategies based on longer-term behavior. A key feature of many RDME models is that this behavior is characterized by a stationary distribution.

To this end, we develop a Gaussian approximation of the output statistics for the RDME model, where the mean of the Gaussian output depends on the equilibrium solution to an underlying deterministic reaction-diffusion model. We rigorously establish conditions for existence of the equilibrium and also show that under our model, the deterministic system converges exponentially fast to the equilibrium.

We numerically validate our theoretical results for several nontrivial chemical reaction-diffusion systems with multiple chemical species. We also show that for many systems of practical interest, the mean of the Gaussian output can be written explicitly in terms of the initial quantities of each chemical species. This forms a rigorous basis to develop new signaling strategies for this inputoutput relationship.

\subsection{Structure of the Paper}

In Section 2, we formalize the model of the reaction-diffusion system and its long-term behavior. In Section 3, we present our approximation for the output statistics, including a detailed study of the 
underlying deterministic model. In Section 4, we numerically validate our approximation. In Section 5, we discuss implications for new signaling schemes and in Section 6, we summarize our results and outline future avenues of research.

\section{SYSTEM MODEL}

Consider a two-dimensional domain ${ }^{1} \Omega \subset \mathbb{R}^{2}$ with smooth boundary $\partial \Omega$ consisting of transmitting and receiving devices, with a fluid medium separating the devices. The domain is discretized into $N$ square voxels of height $h$. Information molecules are generated and received by $K$ first order chemical reactions involving $L$ chemical species $S_{1}, \ldots, S_{L}$ of the form

$$
\mathrm{S}_{l} \rightarrow \mathrm{S}_{n}
$$

Each chemical species $S_{l}, l=1, \ldots, L$ is also associated with a diffusion coefficient $D_{l}(\mathbf{x}) \geq 0, \mathbf{x} \in \Omega$, which is in general spatially inhomogeneous. That is, the diffusion coefficient depends on the location $\mathbf{x}$ in $\Omega$.

The state of the system is defined by the number of molecules for each species, in each voxel. Let $M_{i}^{l}(t), l=1, \ldots, L, i=1, \ldots, N$ denote the random variable for the number of particles of species $S_{l}$ in voxel $j$ at time $t$. Denote $\mathbf{M}_{i}=\left[M_{i}^{1}, \ldots, M_{i}^{L}\right]^{T}$ as the state vector in voxel $i$ and the matrix $\mathbf{M}(t)=\left[\mathbf{M}_{1}, \ldots, \mathbf{M}_{N}\right]$. The probability that $\mathbf{M}(t)$ has value $\mathbf{m}$ at time $t$ is then denoted by

$$
P(\mathbf{m}, t)=\operatorname{Pr}\left(\mathbf{M}(t)=\mathbf{m} \mid \mathbf{M}(0)=\mathbf{m}_{0}\right),
$$

where $\mathbf{M}(0)$ is the initial quantity of molecules in each voxel for each chemical species.

Since each reaction is first-order, it follows that in each reaction the number of molecules for the two species involved can only increase or decrease by one. Let $1_{i}^{l}$ be the state where the number of molecules at all locations is zero, except for species $l$ in voxel $i$. That is, $\mathbf{M}(t)+\mathbf{1}_{i}^{l}$ means that the number of molecules of species $l$ in voxel $i$ is incremented by one.

In this paper, we assume that the system is governed by the RDME. In this model, the diffusive jump rate is denoted by $\kappa_{i j}^{l}$ for each individual molecule of the $l$-th species moving from voxel $j$ into voxel $i$. In particular, the probability per unit time that a molecule of $\mathrm{S}_{l}$ diffuses from voxel $j$ to voxel $i$ at time $t$ is given by $\kappa_{i j}^{l} M_{j}^{l}(t)$.

In the case of mass-action kinetics and first-order reactions, the probability per unit time that a molecule of $S_{l}$ in voxel $i$ reacts to form $S_{n}$ at time $t$ is given by $a_{n l} M_{i}^{l}(t)$ with rate constant $a_{n l}$. Equivalently, for the $k$-th reaction $(k=1, \ldots, K)$, rate constant is denoted by $a^{k}$ and the reaction rate in voxel $i$ is given by $a^{k}\left(\mathbf{m}_{i}\right)$. Each rate constant is assumed to not depend on the voxel index. Further, the net change of each chemical species due to reaction $k$ is given by the column vector $v_{k} \in \mathbb{N}^{L}$. The $L$-dimensional row vector $\mathbf{e}_{i}$ denotes the vector consisting of zeros except for the $i$-th element.

The probability distribution $P(\mathbf{m}, t)$ for the system state then evolves according to the system of differential equations given by

\footnotetext{
${ }^{1}$ We focus on the case of $\Omega \subset \mathbb{R}^{2}$ for concreteness. In fact, many of the results also apply for $\Omega \subset \mathbb{R}^{d}$ for $d \geq 3$. We leave a complete discussion of this scenario for future work.
}

the RDME [11]

$$
\begin{aligned}
\frac{d P(\mathbf{m}, t)}{d t} & =\sum_{i=1}^{N} \sum_{j=1}^{N} \sum_{l=1}^{L}\left(\kappa_{i j}^{l}\left(m_{j}^{l}+1\right) P\left(\mathbf{m}+\mathbf{1}_{j}^{l}-\mathbf{1}_{i}^{l}, t\right)\right. \\
& \left.-\kappa_{j i}^{l} m_{i}^{l} P(\mathbf{m}, t)\right) \\
& +\sum_{i=1}^{N} \sum_{k=1}^{K}\left(a^{k}\left(\mathbf{m}_{i}-v_{k}\right) P\left(\mathbf{m}-\mathbf{e}_{i} \boldsymbol{v}_{k}, t\right)\right. \\
& \left.-a^{k} \mathbf{m}_{i} P(\mathbf{m}, t)\right) .
\end{aligned}
$$

In fact, the RDME corresponds to the Kolmogorov forward equation for a continuous-time Markov chain. That is, the evolution of the system state is Markovian.

In the case that the continuous-time Markov chain corresponding to the RDME is irreducible and positive recurrent, it admits a stationary distribution $\pi(\mathbf{m})$ [13]. In particular, when the stationary distribution exists it is given by

$$
\pi(\mathbf{m})=\lim _{t \rightarrow \infty} \operatorname{Pr}\left(\mathbf{M}(t)=\mathbf{m} \mid \mathbf{M}(0)=\mathbf{m}_{0}\right)
$$

As a consequence, the stationary distribution captures the longterm behavior of the system. In the following section, we study the stationary distribution and show that it can be well-approximated via a simplified Gaussian model. This provides a means of characterizing the input-output relationship of the stochastic chemical reaction-diffusion system, which forms a basis to develop new signaling strategies with reduced complexity based on the long-term behavior.

\section{INPUT-OUTPUT RELATIONSHIP}

In this section, we study the behavior of the output statistics from the stochastic reaction-diffusion system. In particular, we first develop a Gaussian approximation based on a linear noise model. We then exploit this result to characterize the stationary distribution, drawing on techniques from the study of deterministic chemical reaction-diffusion systems.

\subsection{Stochastic Linear Noise Approximation}

It is known that for stochastic chemical reaction networks under mass-action kinetics, the evolution of the molecular counts of each species can be approximated by the chemical Langevin equation [9]. Since diffusion is modeled by a first-order reaction in the RDME model, it follows that a similar approximation can be applied. In particular, we have

$$
\begin{aligned}
& M_{i}^{l}(t+\tau) \\
& \approx M_{i}^{l}(t)+\sum_{j=1}^{N} \kappa_{i j}^{l} M_{j}^{l}(t) \tau+\sum_{j=1}^{N} \sqrt{\kappa_{i j}^{l} M_{j}^{l}(t) \tau} \mathcal{N}_{D, j}(0,1) \\
& +\sum_{k=1}^{K} a^{k}\left(\mathbf{M}_{i}(t)\right) v_{k, l} \tau+\sum_{k=1}^{K} \sqrt{a^{k}\left(\mathbf{M}_{i}(t)\right) v_{k, l} \tau} \mathcal{N}_{R, k}(0,1),
\end{aligned}
$$

where each standard normal random variable $\mathcal{N}_{D, j}(0,1)$ and $\mathcal{N}_{R, k}(0,1)$ is independent. 
Let $V=V_{i}, i=1, \ldots, N$ be the volume of voxel $i$ and define the concentration of species $\mathrm{S}_{l}$ in voxel $i$ by

$$
C_{i}^{l}(t)=\frac{M_{i}^{l}(t)}{V} .
$$

It then follows from the RDME that

$$
\begin{aligned}
\frac{d \mathbb{E}\left[C_{i}^{l}(t)\right]}{d t} & =\sum_{j=1}^{N}\left(\kappa_{i j}^{l} \mathbb{E}\left[C_{j}^{l}(t)\right]-\kappa_{j i}^{l} \mathbb{E}\left[C_{i}^{l}(t)\right]\right) \\
& +\sum_{k=1}^{K} v_{k}^{l} \mathbb{E}\left[a^{k}\left(\mathbf{C}_{i}(t)\right)\right] .
\end{aligned}
$$

Since all reactions are first-order, under the assumption that the diffusion jump rates, $\kappa_{i j}$, are chosen appropriately, the expected concentrations converge to a deterministic reaction-diffusion system in the continuum limit [11]. In particular, let $u_{1}, \ldots, u_{L}$ be the deterministic concentrations. Then, the deterministic system is described by the system of partial differential equations [7], for all $l=1, \ldots, L$,

$$
\begin{cases}\partial_{t} u_{l}-\operatorname{div}\left(D_{l}(\mathbf{x}) \nabla u_{l}\right)=\sum_{n=1}^{L} a_{l n} u_{n}, & \mathbf{x} \in \Omega, \\ D_{l}(\mathbf{x}) \nabla u_{l} \cdot v=0, & \mathbf{x} \in \partial \Omega, \\ u_{l}(\mathbf{x}, 0)=u_{l 0}(\mathbf{x}), & \mathbf{x} \in \Omega,\end{cases}
$$

where the self-reaction rate $a_{l l}$ is defined by

$$
a_{l l}=-\sum_{n=1, n \neq l}^{L} a_{n l} \text {. }
$$

The vector-valued function $v(\mathbf{x})$ is the outer unit normal defined for $\mathbf{x} \in \partial \Omega$. This corresponds to a homogeneous Neumann boundary condition and nonnegative initial data. Recalling that the diffusion coefficients satisfy $D_{l}(\mathbf{x}) \geq 0$ and can be zero on a set with positive measure.

Using the same argument as [1], it follows from the Langevin approximation in (5) that fluctuations are of the order of $\sqrt{V}$. This suggests the Gaussian approximation

$$
M_{i}^{l}(t) \approx V u_{l}\left(\mathbf{x}_{i}, t\right)+\sqrt{V} Z_{i}^{l}(t)
$$

where $Z_{i}^{l}(t)$ is a zero-mean Gaussian random variable and $\mathbf{x}_{i}$ is a point inside the $i$-th voxel. We note that this approximation can be rigorously justified in the case of chemical reaction networks [1] via a convergence result in [6]. To the best of the authors' knowledge, a completely rigorous treatment in the presence of diffusion is not presently available due to the need to account for the continuum limit.

The expression in (9) corresponds to an approximation for the output statistics of the RDME. As such, to provide a complete characterization of the input-output relationship, we require an expression for $u_{l}, l=1, \ldots, L$. In general, $u_{l}$ can only be computed numerically (as will be investigated further in Section 4). Nevertheless, when an equilibrium for the chemical-reaction diffusion system in (1) exists, a simpler representation for $u_{l}$ is available. We study this representation in the sequel.

\subsection{Deterministic Equilibrium Characterization}

The first question in characterizing the deterministic concentrations $u_{l}(\mathbf{x}, t)$ is whether a solution exists. In the case of chemical reaction networks, this question has been extensively studied. In general, the system of partial differential equations in (8) does not have classical solutions due to the possible degeneracy of the diffusion coefficients $D_{l}(\mathbf{x})$. As a consequence, it is necessary to study weak solutions obtained by integrating the partial differential equations in (8) against test functions. The interested reader is referred to [7] for more details.

The following theorem establishing the global existence of weak solutions has been recently established in [7].

THeOREm 3.1. For any nonnegative initial data $\mathbf{u}_{0} \in L^{2}(\Omega)^{L}$, the system (8) has a unique nonnegative weak solution $\mathbf{u}=\left(u_{1}, \ldots, u_{L}\right) \in$ $\left(C\left([0, T] ; L^{2}(\Omega)\right)\right)^{L}$ for any $T>0$. Moreover, the weak solution satisfies the following mass conservation property

$$
\sum_{l=1}^{L} \int_{\Omega} u_{l}(\mathbf{x}, t) d \mathbf{x}=K_{0}:=\sum_{l=1}^{L} \int_{\Omega} u_{l 0}(\mathbf{x}) d \mathbf{x}
$$

for all $t>0$.

Given that a solution $\mathbf{u}_{l}$ exists (in the weak sense), we now seek to characterize conditions under which an equilibrium exists. That is,

$$
\frac{d u_{l}}{d t} \rightarrow 0, \text { as } t \rightarrow \infty, l=1, \ldots, L,
$$

in a suitable sense. To this end, we require the notion of weakly reversible reaction systems.

Definition 3.2. The reaction system (1) is called weakly reversible, if for any two species $S_{l}$ and $S_{n}$ with $l \neq n$, there exist $S_{l_{1}}, S_{l_{2}}, \ldots, S_{l_{r}}$ for $r \geq 0$ such that

$$
\mathrm{S}_{l} \stackrel{a_{l_{1} l}}{\longrightarrow} \mathrm{S}_{l_{1}} \stackrel{a_{l_{2} l_{1}}}{\longrightarrow} \ldots \stackrel{a_{l_{r} l_{r-1}}}{\longrightarrow} \mathrm{S}_{l_{r}} \stackrel{a_{n l_{r}}}{\longrightarrow} \mathrm{S}_{n},
$$

where all the reaction rate constants are positive.

Roughly speaking, if we regard the reaction system as a directed graph where the species are the vertices and the reactions are directed edges, then the reaction system is weakly reversible if and only if the corresponding directed graph is strongly connected.

The following lemma [7] provides conditions to ensure that the system in (8) admits an equilibrium solution.

Lemma 3.3. Assume that the reaction system (1) is weakly reversible. Then, for any fixed positive initial mass $K_{0}>0$, there exists a unique equilibrium ${ }^{2} \mathbf{u}_{\infty}=\left(u_{l \infty}\right) \in(0, \infty)^{L}$ satisfying

$$
\left\{\begin{array}{l}
\sum_{n=1}^{L} a_{l n} u_{n \infty}=0, \quad \text { for all } \quad l=1, \ldots, L, \\
|\Omega| \sum_{l=1}^{L} u_{l \infty}=K_{0} .
\end{array}\right.
$$

We remark that the equilibrium depends only on the initial mass $K_{0}$ but not on the explicit initial data. This means different initial data sharing the same initial mass give rise to the same equilibrium.

\footnotetext{
${ }^{2}$ Since this is a constant equilibrium, it is the equilibrium of both the reaction and reaction-diffusion systems.
} 


\subsection{Impact of Sampling at a Finite Time}

In practice, it is not possible to observe the equilibrium state of a reaction-diffusion system. To this end, it is important to establish how quickly the system converges to equilibrium. In the following theorem, we show that the convergence is exponential.

Theorem 3.4 (Exponential ConVergence to equilibrium). Fix an arbitrary positive initial mass $K_{0}>0$ and assume that the reaction system (1) is weakly reversible. Further, assume that there exists some $l \in\{1, \ldots, L\}$ such that $D_{l}(\mathbf{x}) \geq \alpha_{i}>0$ for all $\mathbf{x} \in \Omega$. Then, the weak solution in Theorem 3.1 converges exponentially to equilibrium with an explicitly computable rate.

Proof. The proof follows closely the arguments in [7]. The condition $D_{l}(x) \geq \alpha_{l}>0$ is to ensure that at least one species diffuses everywhere, which makes it possible to apply an indirect diffusion effect to obtain exponential convergence to equilibrium. First, define the entropy functional

$$
E[\mathbf{u}]=\sum_{l=1}^{L} \int_{\Omega} \frac{\left|u_{l}-u_{l \infty}\right|^{2}}{u_{l \infty}} d \mathbf{x}
$$

and compute its entropy dissipation (see [7])

$$
\begin{aligned}
& D[\mathbf{u}]=-\frac{d}{d t} E[\mathbf{u}] \\
& =\sum_{l=1}^{L} \int_{\Omega} D_{l}(\mathbf{x}) \frac{\left|\nabla u_{l}\right|^{2}}{u_{l \infty}} d \mathbf{x} \\
& +\sum_{l, n=1 ; l<n}^{L}\left(a_{n l} u_{l \infty}+a_{l n} u_{n \infty}\right) \int_{\Omega}\left(\frac{u_{l}}{u_{l \infty}}-\frac{u_{n}}{u_{n \infty}}\right)^{2} d \mathbf{x} .
\end{aligned}
$$

Now assume without loss of generality that $D_{1}(\mathbf{x}) \geq \alpha_{1}>0$ for all $\mathbf{x} \in \Omega$. Then we can estimate the entropy dissipation by

$$
\begin{aligned}
D[\mathbf{u}] & \geq \alpha_{1} \int_{\Omega} \frac{\left|\nabla u_{1}\right|^{2}}{u_{1 \infty}} d \mathbf{x} \\
& +\sum_{l, n=1 ; l<n}^{L}\left(a_{n l} u_{l \infty}+a_{l n} u_{n \infty}\right) \int_{\Omega}\left(\frac{u_{l}}{u_{l \infty}}-\frac{u_{n}}{u_{n \infty}}\right)^{2} d \mathbf{x} .
\end{aligned}
$$

Using [7, Theorem 2.6] we then have

$$
D[\mathbf{u}] \geq \beta E[\mathbf{u}]
$$

for some $\beta>0$, which can be explicitly computed. It then follows from an application of Gronwall's lemma that

$$
\sum_{l=1}^{L} \int_{\Omega} \frac{\left|u_{l}(t)-u_{l \infty}\right|^{2}}{u_{l \infty}} d \mathbf{x} \leq e^{-\beta t} \sum_{l=1}^{L} \int_{\Omega} \frac{\left|u_{l 0}-u_{l \infty}\right|^{2}}{u_{l \infty}} d \mathbf{x} .
$$

In other words, the trajectory converges exponentially to equilibrium in $L^{2}$-norm as $t \rightarrow \infty$.

A key observation from Theorem 3.4 is that even for finite times, the equilibrium solution for first-order chemical reaction-diffusion systems may form a good approximation of $u_{l}$. In the following section, we validate Theorem 3.4 for the system (8) and show that (9) is a good approximation for the stationary distribution of the RDME model. In Section 5, we discuss implications of these results for the design of molecular communication systems.

\section{NUMERICAL RESULTS}

\subsection{Validation of Exponential Convergence to Equilibrium}

We validate the exponential convergence to equilibrium in Theorem 3.4 in the following two examples. In both examples, we consider multiple first-order reactions and spatially inhomogeneous diffusion coefficients. Note that these systems correspond to the deterministic model in (8) and that we will investigate the behavior of the stochastic model in Section 4.2.

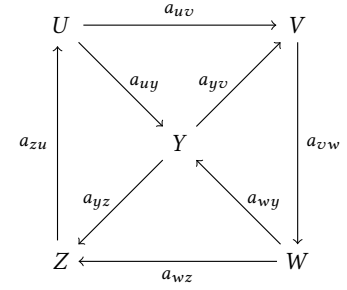

Figure 1: A weakly reversible reaction network with five species.

Our first example considers the reactions in Fig. 1. In this example, the system lies in the unit square $\Omega=(0,1)^{2}$. The sub-domains $\Omega_{1}=(0,1 / 2) \times(0,1)$ and $\Omega_{2}=[1 / 2,1) \times(0,1)$ correspond to a transmitting and receiving devices, respectively.

The diffusion coefficients of the species are $D_{u}(\mathbf{x})=D_{w}(\mathbf{x})=$ $1_{\Omega_{1}}$ and $D_{v}(\mathbf{x})=D_{z}(\mathbf{x})=1_{\Omega_{2}}$, where $1_{X}$ is the indicator function of $X$, i.e. $\mathbf{1}_{X}(\mathbf{x})=1$ if $\mathbf{x} \in X$ and $\mathbf{1}_{X}(\mathbf{x})=0$ otherwise. The diffusion coefficient of $Y$ is given by $D_{y}(\mathbf{x})=\frac{1}{1+|\mathbf{x}|^{2}}$ and we note that $D_{y}(\mathbf{x}) \geq$ $1 / 2$ for all $\mathbf{x} \in \Omega$. That is, only the species $Y$ is able to act as an information molecule, diffusing from the transmitter to the receiver. We note, however, that the reaction rates are spatially homogeneous and therefore the other species may also have nonzero concentrations within the fluid medium.

All the assumptions in Theorem 3.4 are satisfied. As such, the system in Fig. 1 admits exponential convergence to equilibrium. To illustrate the rate of convergence, Fig. 2 plots the concentration trajectories with initial data $u_{0}=0.3 x_{1} 1_{\Omega_{1}}, v_{0}=0.1 x_{2} 1_{\Omega_{2}}$, $w_{0}=0.5 x_{1} 1_{\Omega_{1}}, z_{0}=0.3 x_{2} 1_{\Omega_{2}}, y_{0}=0$, and reaction rate constants $a_{u v}=3, a_{v w}=2, a_{w z}=4, a_{z u}=2, a_{u y}=3, a_{w y}=1$, $a_{y z}=5, a_{y v}=2$. The equilibrium is $\left(u_{\infty}, v_{\infty}, w_{\infty}, z_{\infty}, y_{\infty}\right)=$ $(0.027,0.055,0.022,0.081,0.015)$. 


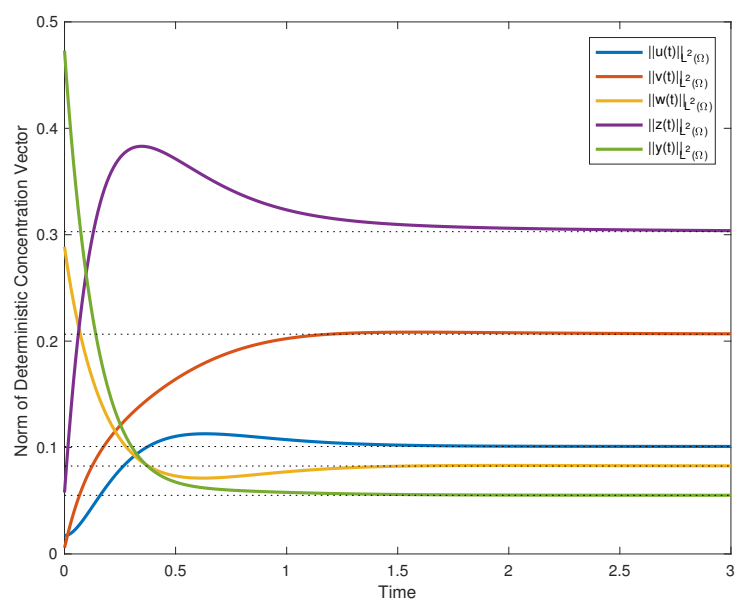

Figure 2: Convergence to equilibrium for the five species system in Fig. 1.

In our second example, we consider the system defined in Fig. 3. In this case, there is a simple closed-form representation of the equilibrium. Set the initial mass $K_{0}:=\int_{\Omega}\left(u_{0}(\mathbf{x})+v_{0}(\mathbf{x})+y_{0}(\mathbf{x})\right) d \mathbf{x}$. By Lemma 3.3

$$
\begin{aligned}
& u_{\infty}=\left(1+\frac{a_{u v}+a_{u y}}{a_{v u}}+\frac{a_{u y}}{a_{y v}}\right)^{-1} \frac{K_{0}}{|\Omega|}, \\
& v_{\infty}=\frac{a_{u v}+a_{u y}}{a_{v u}} u_{\infty}, \quad y_{\infty}=\frac{a_{u y}}{a_{y v}} u_{\infty} .
\end{aligned}
$$

In particular, if $|\Omega|=1$ and all reaction rate constants are equal, we obtain

$$
u_{\infty}=\frac{K_{0}}{4}, \quad v_{\infty}=\frac{K_{0}}{2}, \quad y_{\infty}=\frac{K_{0}}{4} .
$$

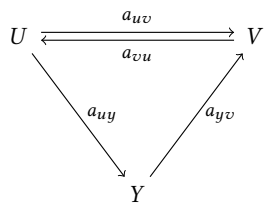

Figure 3: A weakly reversible reaction network with three species.

We demonstrate this case numerically in Fig. 4 where we take $K_{0}=1$ with initial data $u_{0}(\mathbf{x})=x_{1} 1_{\Omega_{1}}, v_{0}(\mathbf{x})=0.5 x_{2} 1_{\Omega_{1}}, y_{0}(\mathbf{x})=0$, domain $\Omega=(0,1)^{2}$, diffusion coefficients $D_{u}=1_{(0,1 / 2) \times(0,1)}, D_{v}=$ $\mathbf{1}_{[1 / 2,1) \times(0,1)}$ and $D_{y}=\frac{1}{1+|\mathbf{x}|^{2}}$.

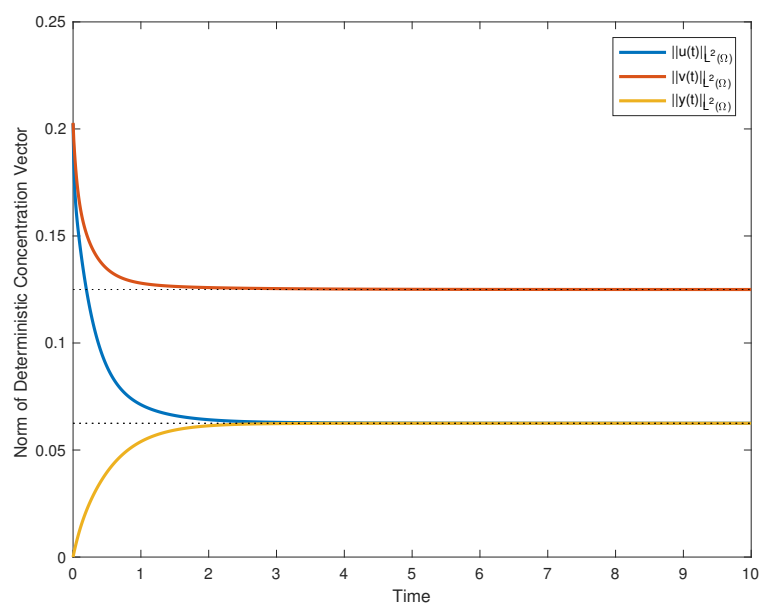

Figure 4: Convergence to equilibrium for the three species system in Fig. 3.

\subsection{Validation of the Input-Output Relationship in (9)}

To validate our input-output relationship in (9), we consider the two species system consisting of the reactions

$$
\begin{aligned}
& \mathrm{S}_{1} \stackrel{a_{21}}{\longrightarrow} \mathrm{S}_{2} \\
& \mathrm{~S}_{2} \stackrel{a_{12}}{\longrightarrow} \mathrm{S}_{1},
\end{aligned}
$$

where $a_{21}=a_{12}=1$. The domain is given by $\Omega=(0,1) \times(0,0.025)$, with the transmitter located in $\Omega_{1}=(0,0.025) \times(0,0.025)$ and the receiver located in $\Omega_{2}=(0.9750,1) \times(0,0.025)$. The diffusion coefficients are given by $D_{1}(\mathbf{x})=\mathbf{1}_{(0,0.025) \times(0,0.025) \cup(0.9750,1) \times(0,0.025)}$ (corresponding to $S_{1}$ ) and $D_{\mathrm{S}_{2}}(\mathbf{x})=1$ (corresponding to $\mathrm{S}_{2}$ ). The initial data is $u_{10}(\mathbf{x})=u_{20}(\mathbf{x})=\frac{100}{0.025^{2}} \mathbf{1}_{(0,0.025) \times(0,0.025)}$ corresponding to 100 molecules of $S_{1}$ and $S_{2}$ in the transmitter and receiver, respectively.

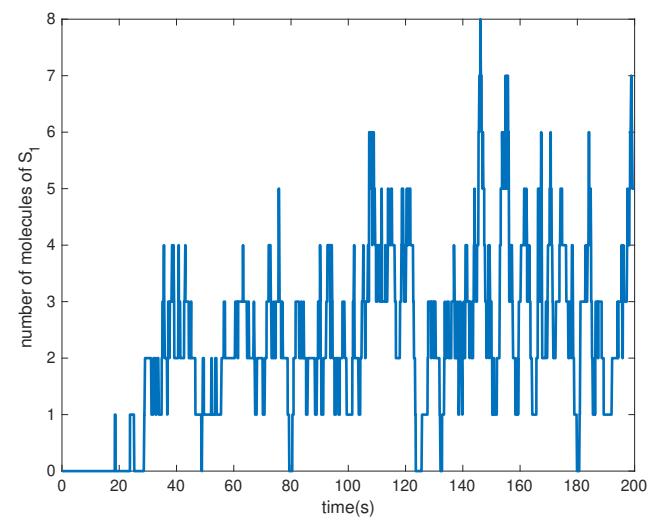

Figure 5: Realization of the process for the number of molecules of $S_{1}$ in the receiver voxel. 


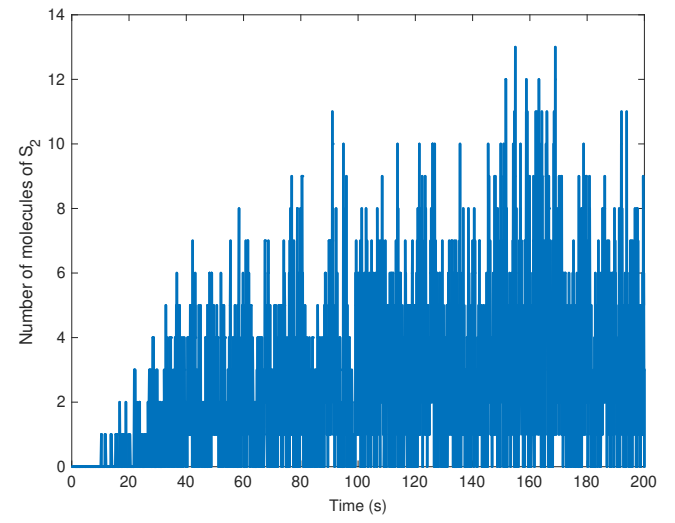

Figure 6: Realization of the process for the number of molecules of $S_{2}$ in the receiver voxel.

Figures 5 and 6 plot a realization of the process for the number of molecules of $S_{1}$ and $S_{2}$ respectively. To verify (9), we compute the mean number of molecules of $S_{1}$ and $S_{2}$, which is found to be approximately 2.5 based on the last 100 samples. Note that this is consistent with the deterministic system as by Lemma 3.3, the concentrations are spatially homogeneous with the same values for $\mathrm{S}_{1}$ and $\mathrm{S}_{2}$. In particular, since the initial mass is 100 for each species, it follows that the approximate mean is $\frac{100}{40}=2.5$, consistent with the stochastic simulation.

\section{DISCUSSION}

In Section 3, we provided a characterization of the output statistics for a molecular communication system modeled via the RDME. In particular, for sufficiently large sampling times, we have shown that the output is well-approximated by a Gaussian random variable with mean given by the equilibrium solution to the underlying deterministic chemical reaction-diffusion system.

As a consequence, this work provides initial results towards a rigorous foundation for equilibrium signaling schemes. In these schemes, first studied without the presence of diffusion in [5], the receiver decodes information based on the estimation of the mean number of molecules for a given chemical species. In the case that the equilibrium solution for the underlying deterministic reactiondiffusion system can be easily characterized in terms of the initial conditions (i.e., the number of molecules initially produced within the transmitter), the receiver architecture is simplified and more general continuous-time filtering methods (e.g., [4]) are not required.

Nevertheless, for equilibrium signaling schemes to be widely applicable, it is necessary to obtain simple representations for the equilibrium solutions in the deterministic model detailed in (8). To this end, we note that under the conditions in Theorem 3.4, the equilibrium solutions are spatially homogeneous. In particular, these solutions are given by Lemma 3.3. In general, the solutions arising from Lemma 3.3 depend on the reaction coefficients. As noted in [5], under particular structures explicit solutions can be obtained from advection dynamics [2]. A simple example arises in the case that all reaction coefficients are the same [5] or the choice in the second example in Section 4.1.

\section{CONCLUSION}

In this paper, we studied the input-output relationship for a general class of systems modeled by the reaction-diffusion master equation. In particular, we obtained a Gaussian approximation for the output statistics, validated via stochastic simulation.

Our approximation is based on a link to the equilibrium solutions of the underlying deterministic reaction-diffusion system, described by a system of partial differential equations. Solutions for this system have only recently been characterized and by exploiting the methods in [7], we established exponential convergence to equilibrium for the case of inhomogeneous diffusion coefficients. This provides insights into the finite-time behavior of the system.

A number of open issues still remain. Our approximation, motivated by a Langevin equation for the system, has only been rigorously established in the case of chemical reaction networks without diffusion. Therefore, we intend to study the rigorous treatment of the reaction-diffusion scenario. Moreover, extending the results to inhomogeneous reaction rate coefficients is also an open problem. Another avenue is to exploit our characterization to develop new signaling schemes, generalizing the work in [5].

\section{ACKNOWLEDGEMENTS}

Bao Q. Tang is partially supported by NAWI Graz and the International Training Program IGDK 174.

\section{REFERENCES}

[1] L. Cardelli, M. Kwiatkowska, and L. Laurenti. 2016. Stochastic analysis of chemical reaction networks using linear noise approximation. BioSystems 149 (2016), 2633.

[2] A. Chapman and M. Mesbahi. 2011. Advection on graphs. In IEEE Conference on Decision and Control.

[3] C.T. Chou. 2013. Extended master equation models for molecular communication networks. IEEE Transactions on NanoBioscience 12, 2 (2013), 79-92.

[4] C.T. Chou. 2015. A Markovian approach to the optimal demodulation of diffusionbased molecular communication networks. IEEE Transactions on Communications 63, 10 (2015), 3728-3743.

[5] M.Egan, T.C. Mai, T.Q. Duong, and M. Di Renzo. 2018. Coordination via advection dynamics in nano networks with molecular communication. In IEEE International Conference on Communications (ICC).

[6] S.N. Ethier and T.G. Kurtz. 2009. Markov Processes: Characterization and Convergence. John Wiley \& Sons.

[7] K. Fellner, W. Prager, and B. Tang. 2017. The entropy method for reaction-diffusion systems without detailed balance: first order chemical reaction networks. Kinetic and Related Models 10, 4 (2017), 1055-1087.

[8] D. Gillespie. 1977. Exact stochastic simulation of coupled chemical reactions. The Journal of Physical Chemistry 81, 25 (1977), 2340-2361.

[9] D.T. Gillespie. 2009. The deterministic limit of stochastic chemical kinetics. $f$ Phys Chem B 113, 6 (2009), 1640-1644.

[10] A. Hamdan and C.T. Chou. 2017. Generalized solution for the demodulation of reaction shift keying signals in molecular communication networks. IEEE Transactions on Communications 65, 2 (2017), 715-727.

[11] S.A. Isaacson. 2009. The reaction-diffusion master equation as an asymptotic approximation of diffusion to a small target. SIAM f. Appl. Math. 70, 1 (2009), 77-111.

[12] A. Noel, K. Cheung, and R. Schober. 2014. Improving receiver performance of diffusive molecular communication with enzymes. IEEE Transactions on NanoBioscience 13, 1 (2014), 31-43.

[13] J.R. Norris. 1998. Markov chains. Cambridge University Press.

[14] E. Shitiri, A. Vasilakos, and H.-S. Cho. 2018. Biological Oscillators in Nanonetworks-Opportunities and Challenges. Sensors 18, 5 (2018), 1544. 